\title{
MInOSSE: A new method to reconstruct geographic ranges of fossil species
}

\author{
Francesco Carotenuto $^{1}$ (D) | Mirko Di Febbraro ${ }^{2}$ (D) | Alessandro Mondanaro ${ }^{3}$ | \\ Silvia Castiglione $^{1}$ | Carmela Serio ${ }^{1}$ | Marina Melchionna ${ }^{1}$ | Lorenzo Rook $^{3}$ (D) | \\ Pasquale Raia ${ }^{1}$
}

\author{
${ }^{1}$ Dipartimento di Scienze della Terra, \\ dell'Ambiente e delle Risorse, Università \\ degli Studi di Napoli Federico, Napoli, Italy \\ ${ }^{2}$ Dipartimento di Bioscienze e Territorio, \\ University of Molise, Campobasso, Italy \\ ${ }^{3}$ Dipartimento di Scienze della Terra, \\ Università di Firenze, Firenze, Italy

\section{Correspondence} \\ Francesco Carotenuto \\ Email: francesco.carotenuto@unina.it \\ Handling Editor: Huijie Qiao
}

\begin{abstract}
1. Estimating fossil species' geographic range is a major goal for paleobiologists. In the deep time, this is most commonly performed by using polygon-based methods such as the minimum convex polygon (MCP) or the Alpha-Hull. Unfortunately, such methods provide a poor representation of the fossil species' actual range, because they are unable to take control of the severe stochastic and taphonomic biases.
\end{abstract}

2. Here, we introduce MInOSSE (massively interpolated occurrences for species spatial estimation), a model-based method that combines a machine learning algorithm and geostatistical approaches to reconstruct a target fossil species' geographic ranges by relying on the distribution of other coeval species and without using environmental predictors.

3. We tested MInOSSE by using many simulated fossil species' distributions, comparing its performance with MCP and Alpha-Hull outcomes and applying it to real case studies.

4. In all simulations, MInOSSE outperformed the competing methods. Interestingly, the superior performance of MInOSSE becomes more apparent when the fossil record of the target species is scarce, that is, when appropriate range reconstruction is most problematic with polygon-based methods. updates
updater 
commonly applied than AOOs, and usually estimated by means of minimum convex polygon (MCP) or Alpha-Hull. Such polygon-based methods are quick and simple to apply as they require either a single (Alpha-Hull) or no (MCP) parameter to tune. Yet, they often provide a poor representation of the actual species' spatial distribution, since they lack any predictive power outside their geometry and the number of reconstructed disjunct patches depends on parameter setting. In the case of fossil species, the limitations of polygon-based methods worsen. Indeed, in terms of reconstructing fossil species' geographic ranges, geological and taphonomic processes could affect the quality of paleontological information if not properly accounted for (Noto, 2011; Plotnick, 2017; Raia et al., 2009). However, polygon-based methods, as well as other geodesic approaches like latitudinal range and maximum circle distance, proved to be very accurate in providing relative range size estimates and dynamics (Darroch \& Saupe, 2018), despite their poor performance in spatial reconstruction.

As compared to polygon-based methods, model-based approaches provide more realistic range reconstructions, predicting species' occurrence at unsampled sites regardless of the nature and age of the outcrops and of sampling intensity. Species distribution models (SDMs) have been thus successfully applied to fossil species (Carotenuto et al., 2016; Di Febbraro et al., 2017; Melchionna et al., 2018; Nogués-Bravo, Rodríguez, Hortal, Batra, \& Araújo, 2008; Svenning, Fløjgaard, Marske, Nógues-Bravo, \& Normand, 2011; Varela, Lobo, \& Hortal, 2011). Unfortunately, SDMs need spatially explicit environmental variables, which are just available for the last few kiloyears (Friedrich et al., 2010; Singarayer \& Valdes, 2010). Consequently, deep-time macroecology cannot make any use of SDMs due to the lack of suitable environmental predictors.

Ideally, the geographic distribution of a group of species should be informative about the probability for a given species to occur in a certain place (Gotelli \& McCabe, 2002). At least in theory, such distributions could be used as predictors in a model-based approach to calculate the occurrence probability of just one species, as well as its geographic range size, shape and position. Here, we introduce MInOSSE (massively interpolated occurrences for species spatial estimation), a model-based method to reconstruct the geographic ranges of fossil species (the target) by using the occurrences of its coeval species (the predictors) for temporal intervals when no other environmental data are available. According to the theory of 'taphonomic control' (sensu Bottjer, Droser, \& Jablonski, 1988), in a stable fossil community, the occurrence of some species can be indirect evidence of another species' presence or absence. By relying on coeval species used as predictors, MInOSSE can be less affected than polygons-based methods by the patchy nature of the fossil record in reconstructing the spatial distribution of the target. First, our method reconstructs the predictor species' distributions by means of geostatistical technique, and then the geographic range of the target one by combining the target's record and the predictors in a machine learning algorithm. We performed stochastic and biologically driven simulations based upon real fossil data to test the performance of MInOSSE in predicting the actual distributions of simulated fossil species and compared its performance with MCP and the Alpha-Hull outcomes. Tests of MInOSSE's performance were performed on living species after applying an artificial process of fossilization. We then show case studies with real fossil record.

\section{2 | MATERIALS AND METHODS}

MInOSSE consists of two main algorithms. The first function (minosse. data) takes a dataset with species' names and their individual fossil occurrences (including estimated mean age and geographical coordinates). Given a target species, whose range is to be estimated, minosse.data keeps the fossil occurrences of the target apart from the fossil record and generates the spatial distributions of all the species coeval to the target by means of spatial interpolation. The second function (minosse. target) predicts the spatial distribution of the target by performing Regression Kriging over the minosse.data output. A schematic explanation of these algorithms is summarized in Figures 1 and 2, respectively.

\section{1 | minosse.data. Generating predictor variables}

\subsection{1 | Input data}

The first step to run minosse.data consists of providing a dataset containing the fossil species' occurrences supplied with their geographic coordinates, the related age estimates and fossil locality identifiers. This dataset must include both the target and its coeval (the predictors) species' occurrences. The user can specify a spatial domain where MInOSSE will reconstruct the target species' geographic range. The spatial domain is provided in the form of a polygon shapefile or a raster map. This file is automatically converted into a spatial grid with a predetermined resolution (see below), which is used to simulate species' pseudo-absences and to perform spatial interpolations. The users may provide their own paleo maps of the world to define spatial domain, once the geographical coordinates of fossil localities are properly converted to paleo-coordinates.

Once the fossil dataset is provided, by default MInOSSE automatically detects the species whose temporal range covers the target species' 95\% time span (although alternative temporal overlaps can be provided). Then, it builds an occurrence matrix by assigning the value 1 to all the fossil sites where a species is present. Species' absences in the paleontological sites are discarded as it is not possible to distinguish between real absences (structural zeroes) and sampling biases. Therefore, predictor species' pseudo-absences have to be simulated. Under MInOSSE, this is done by either of two strategies. The simplest consists of specifying a predetermined number of pseudo-absences for all predictors. The second strategy sets the number of pseudo-absences for each predictor species to be equal to the species' presences. The later criterion corresponds to the so-called 'D-optimal design', which states half of the observations must be at one extreme ( 1 scores) and the remaining at the other extreme (0 scores), in order to minimize the prediction variance (Montgomery, 2004). Having set a spatial 


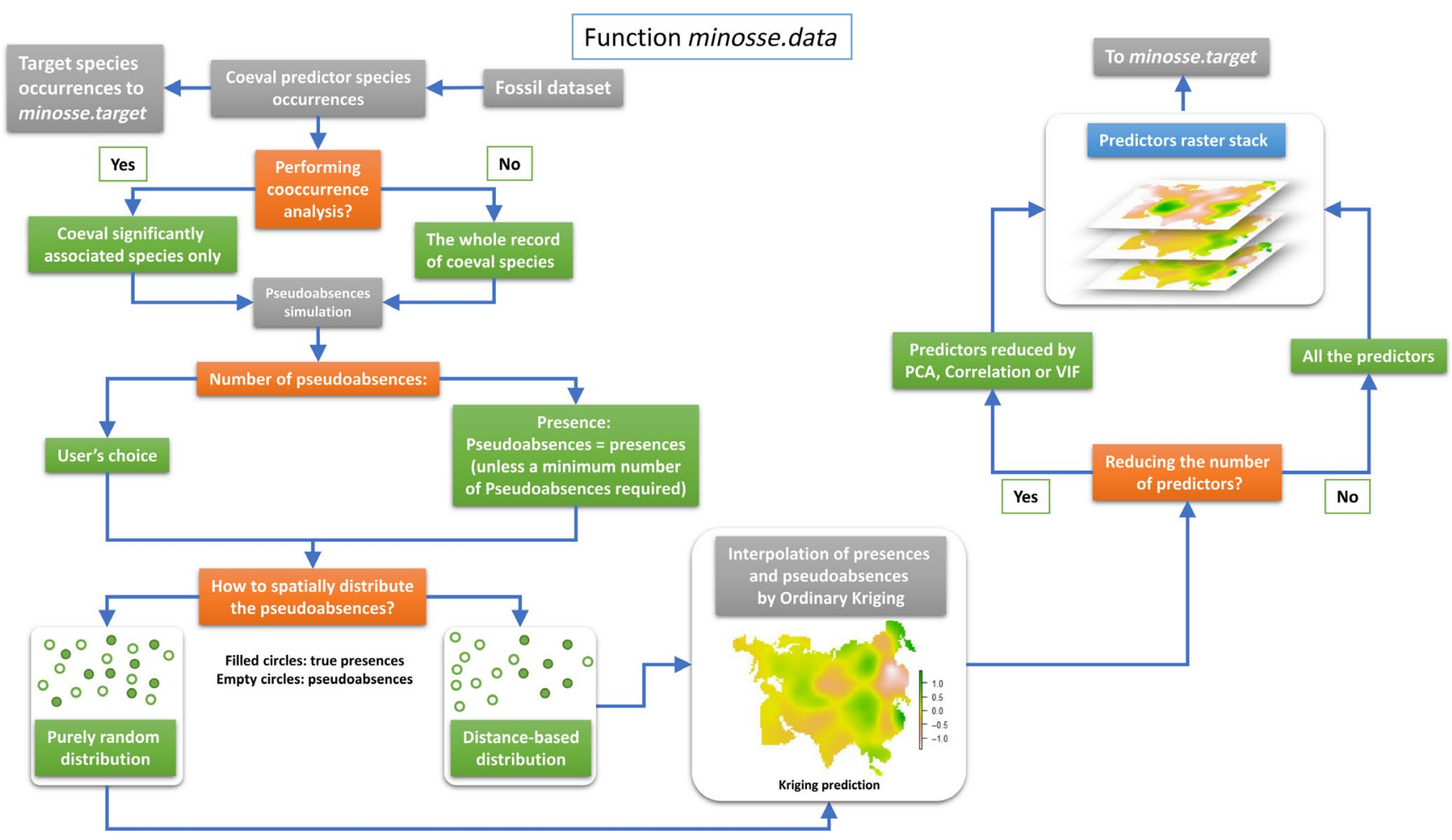

FIGURE 1 Flow chart showing the minosse.data algorithm (see the main text for detailed explanations of individual steps)

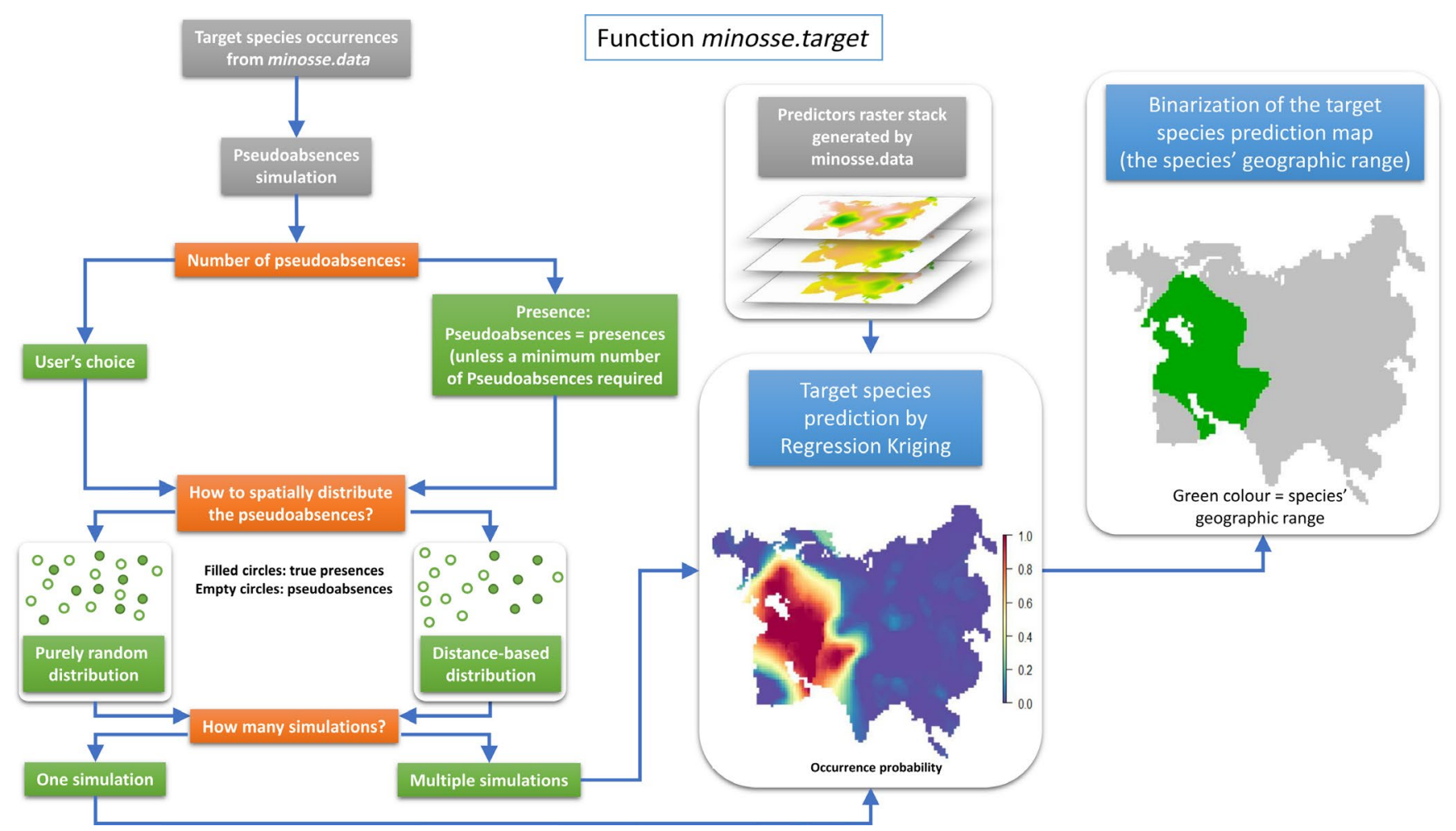

FIGURE 2 Flow chart showing the minosse.target algorithm (see the main text for detailed explanations of individual steps)

domain, the pseudo-absences for each species are located according to either a pure, random distribution, or a distance-based distribution, avoiding cells where a species occurs. Under the latter distribution, the probability of a pseudo-absence to occur in a given place is inversely proportional to the distance from actual presences, under the assumption that actual presences represent 
an unbiased sub-sample of the species' natural distribution (Hengl, Sierdsema, Radović, \& Dilo, 2009).

\subsection{2 | Spatial interpolation}

The spatial interpolation between presences (1s) and pseudoabsences (Os) is performed for each predictor by means of Ordinary Kriging, as implemented in R package INTAMAP (Pebesma et al., 2011). This algorithm provides solutions to interpolate variables with a nonGaussian distribution, automated variogram modelling and checking for anisotropy in the spatial distribution of the variables (Pebesma et al., 2011). The output of each interpolation consists in a raster map showing the predictor species' distribution probability (see Appendix 1 in Supporting Information for details about spatial interpolation). To properly build a semivariogram and to perform Kriging interpolation, a minimum of 30-point locations is required with no distinction between presences and pseudo-absences. Since this condition might not be met by all predictors, the user is allowed to set a minimum required number of pseudo-absences to be simulated.

\subsection{3 | Reduction of predictors dimensionality}

Under MInOSSE, species that are coeval to the target are used as predictors. However, not all predictors are informative. Predictors redundancy inflates variance and makes the whole algorithm timeconsuming. MInOSSE uses two different strategies to select informative predictors. First, it may apply co-occurrence analysis to the species' lists, and select only predictors that are significantly associated to the target. This analysis assigns to any possible combination of species pairs, for instance the species $A$ and $B$, a co-occurrence probability by computing the chance of randomly selecting (with replacement) a fossil site that has the species $A$ given that it already has species $B$. In MInOSSE, the unit of co-occurrence is either the species' list of each fossil site or the list of species falling in the same geographic cell. Co-occurrence analysis is performed by using the R package cooccur (Griffith, Veech, \& Marsh, 2016). The second strategy to select predictors is to compute their distribution probability maps derived by spatial interpolations and calculate either Pearson's correlation, Variance Inflation Factor or Principal Component Analyses to drop correlated predictors. These two strategies are not mutually exclusive (see Appendix 1 in Supporting Information for details about predictors dimensionality reduction).

\section{2 minosse.target. Predicting the target (response) species' geographic range}

\subsection{1 | Input data}

The simulation of the target species' pseudo-absences takes place in this step and is performed as for the predictors. In addition, MInOSSE allows to generate multiple replicates of pseudo-absences, each one used to draw a specific map of the target species' distribution. These maps are then averaged to produce a final distribution map including the contribution of all the pseudo-absences simulations. Combining multiple target species' distribution maps complies with the findings of Barbet-Massin, Jiguet, Albert, and Thuiller (2012), who suggested this is the optimal protocol to perform Boosted Machine Learningbased species' distribution modelling.

\subsection{2 | Regression Kriging}

The target species' distribution is predicted by means of Regression Kriging (RK, Hengl, Heuvelink, \& Rossiter, 2007). This technique combines the information derived by a deterministic description of the spatial variability of a variable (a machine learning algorithm, MLA) and the interpolation of the MLA residuals via Kriging to take into account the stochastic component of variability (Hengl et al., 2007). The species' distribution is generated by the MLA first, then the model's prediction errors are interpolated via Kriging and used to update a new MLA. This provides a prediction that accounts for spatial autocorrelations between the observations. In MInOSSE, the use of RK is crucial since the predictor species' maps are generated via Kriging interpolation, which produces spatial autocorrelation between the probability values at unsampled locations, increasing model stochasticity. A general overview of RK algorithm is provided in Appendix 2 of Supporting Information.

We used the XGBoost (Chen \& Guestrin, 2016; Appendix 2 of Supporting Information) as the MLA producing the deterministic part of RK, as in Hengl et al. (2017). MLAs do not need any specific assumptions about the response variable for linear prediction and provide the best fit for complex non-linear relationships between multiple predictors (Hengl et al., 2015, 2017). The RK procedure uses functions embedded in the R package GSIF (Hengl, 2020). RK prediction maps are binarized to draw species' geographic range by applying the following nine threshold metrics: 'Sens = Spec' (a threshold where sensitivity equals specificity), 'MaxSens + Spec' (a threshold that maximizes the sum of sensitivity and specificity and that is equivalent to Youden's J statistic; Youden, 1950), 'MaxKappa' (a threshold that gives the maximum value of Kappa), 'MaxPCC' (which maximizes the total accuracy), 'PredPrev = Obs' (predicted prevalence is set equal to the observed prevalence), 'ObsPrev' (a threshold equal to the observed prevalence), 'MeanProb' (equivalent to the mean probability of occurrence yielded by the model), 'MinROCdist' (a threshold that minimizes the distance between the ROC plot and the upper left corner of the unit square), 'Cost' (which balances the relative costs of false positive predictions and false negative predictions). All the described thresholds are computed by using the R CRAN package PresenceAbsence (Freeman \& Moisen, 2008). RK needs 50 unique point locations at least (including both presences and pseudo-absences) to perform the internal cross validation procedure as provided in the GSIF package. In MInOSSE, the user has the option to simulate as many pseudo-absences as the presences and 
specify a minimum required number of pseudo-absences in order to perform RK.

\section{3 | Simulation experiments}

To test MInOSSE, we compared its results with the actual geographic distributions of simulated fossil species. We built two different simulation sets: Eco-sims, that is principally used to measure the MInOSSE performance under different parameters' settings; and ST-sims, whose main goal is to assess MInOSSE under different spatial distribution simulations. Under both Eco-sims and ST-sims, the fossil records are simulated as follows: (a) create artificial species distributions; (b) draw the actual geographic range for each species by overlaying a cell grid to its occurrences; (c) simulate a spatially defined pattern of fossil sites inside the geographic domain; (d) extract artificial species lists from the intersection between fossil sites and the actual species ranges; (e) randomly remove occurrences from each list to simulate taphonomic biases. The intensity of the bias is inversely proportional to the geographic range, meaning that rare species are affected by proportionally worse bias (see Appendix 3 in Supporting Information).

The first simulation set, Eco-sim (based on theoretical and ecological processes) works by generating the species' distributions (the step 1 above) through the Artificial Bell Response algorithm of Varela, Anderson, García-Valdés, and Fernández-González (2014) provided in the R package SDMvSPECIES (Duan, Kong, Huang, Wu, \& Wang, 2015). According to this algorithm, the suitability values of a species follow the multivariate normal distribution. The algorithm uses occurrences and bioclimatic variables to produce theoretically based species' distributions. In order to perform realistic simulations, we calibrated the Artificial Bell Response algorithm with the fossil record of Eurasian large mammals living during the last glacial maximum (LGM, from some 26 to some 14 kya) provided in Carotenuto, Diniz-Filho, and Raia (2015) and Carotenuto et al. (2016, 2018). LGM bioclimatic variables were downloaded from www.worldclim. org (Appendix 3 in Supporting Information). For the simulations of the fossil localities, we considered three different scenarios. First, we simulated sites by relying on the spatial pattern of a real fossil record. In detail, we built a density map of the Eurasian large mammalian fossil localities during the LGM and used this spatial layer as probability map to simulate new sites (Appendix 3 in Supporting Information). Although this is the most realistic scenario, simulated species and fossil sites might not be totally independent from each other since they were generated by relying on the same real fossil record. Hence, we considered two additional stochastic scenarios: one in which we simulated spatially high-clustered fossil localities and a last one with randomly distributed sites. In this way, we ensured species and fossil localities to be totally independent simulated data. For parameter settings, we used this set of simulations to: (a) compare MInOSSE predictions with and without preliminary co-occurrence analysis by means of Permutational MANOVA (see Appendix 4); (b) measure the impact of different grid resolutions for spatial interpolation on MInOSSE predictive accuracy; and (c) compare MInOSSE with traditional polygon-based methods. For (b), we used square cell of 100,200 , or $300 \mathrm{~km}$ side. A fourth and fifth cell resolution levels have the side of the cell equal to the mean nearest neighbour distance between fossil sites, and half this value. For each of the different MInOSSE parameters setting, we simulated 26 species and 100 fossil localities (that is as many as the number of species in the LGM record we used).

The second simulation set, the ST-sim, is based on a combination of stochastic and theoretically informed processes. Artificial species' occurrences were simulated according to a core-periphery abundance model (Brown, 1995), which implies a higher occurrence density towards the core and a positive relationship between abundance and geographic range size (Gaston \& Curnutt, 1998). At the interspecific level, the distribution of species' abundances follows the $\log _{2}$-normal (Preston, 1962; see Appendix 3). The simulations are generated to: (a) compare MInOSSE predictions with and without preliminary co-occurrence analysis by means of Permutational MANOVA (Appendix 4); (b) assess whether MInOSSE performance is affected by non-random distribution of occurrence record and fossil localities (i.e. the Wallace shortfall, the process whereby the spatial distribution of information depends on the distribution of the sampling effort or taphonomic bias, Hortal et al., 2015); and (c) compare MInOSSE to traditional polygon-based methods. The distribution of both species occurrence records and fossil localities were based on four different grades of spatial clustering (highly clustered, moderately clustered, random and uniformly distributed). For any single spatial clustering grade, we generated 100 species and repeated the simulations twice, with 100 and 200 fossil localities (Appendix 3 in Supporting Information).

For the Eco-sims, we used the polygon boundaries of the Eurasian continent during the LGM time period, whereas for the ST-sims, we relied on the polygon of present-day Eurasia. For all the simulations, we considered different species' sample sizes, with the minimum set to three occurrences. We run MInOSSE with each of the simulated species and the probability maps were binarized by means of the previously described nine threshold metrics.

\subsection{Comparing MInOSSE with hull methods}

We compared MInOSSE to the MCP and the Alpha-Hull in predicting simulated species' actual geographic ranges. MCP is the smallest convex polygon including all the species occurrences, whereas the Alpha-Hull is the polygon built by point pair connections that can be touched by an empty disc of radius alpha. Alpha is estimated through Maximum Likelihood Estimation as the smallest value providing a single polygon enclosing all the target species' occurrences. To compare the method's performance, we first converted simulated geographic range polygons into presence/absence raster maps having the same resolution of the MInOSSE outputs. Binary maps of the three methods were used to measure their performance in matching the presence/absence maps of the simulated species' actual distributions. The metric for comparison was the 
true skill statistic (TSS; Allouche, Tsoar, \& Kadmon, 2006). TSS is the sum of true positives (i.e. right detected true 1 scores) and true negatives (i.e. right detected true 0 scores) proportions minus 1 (see Appendix 4 for further details about methods' comparisons).

We performed permutational MANOVA with PCA scores of all the binarized MInOSSE maps to measure the method sensitivity to changes in the simulation settings (the number of simulated fossi localities, cell size for Kriging interpolation, binarization thresholds; see Appendix 4 in Supporting Information for detailed explanations about all these tests). Eventually, we compared the TSS scores by means of ANOVA and Tukey Honest Significant Differences (HSD) post-hoc test using the three methods as factors. We did not consider the geographical domain of the whole simulated fossil record for TSS computation, but rather used the territories bounded by the rectangle enclosing the simulated species' actual geographic range only, although this restriction penalizes the specificity of MInOSSE.

\subsection{Application of MInOSSE to real case studies}

We used MInOSSE to reconstruct the geographic range of the woolly mammoth Mammuthus primigenius. This iconic Ice Age species was adapted to tundra/steppe biomes and inhabited vast territories from the Western to the Eastern Eurasia up to North America (Kahlke, 2015). Its abundance and geographic range widened during the LGM (since 24 to some 14 kya), then going extinct during the Early/Mid Holocene, when its habitat dwindled rapidly following the post-LGM global warming. We used the dataset published in Carotenuto et al. (2015, 2018), restricting the fossil record to the 24-14 kya time frame, that is, the period of maximum geographic expansion for M. primigenius. Therefore, we predicted MInOSSE to draw a single, contiguous and large geographic range for the species, extending over Central to Northern Eurasia, that is, the so-called Mammoth steppe. This steppe biome seamlessly extended from Kamchatka to Northern Iberia during LGM (Guthrie, 1982).

We further used MInOSSE to estimate the geographic range of the roe deer Capreolus capreolus from 130 kya to the recent. There has been much debate about the phylogenetic and taxonomic position of this species. Once considered a single species covering most of the Eurasia, it is now widely accepted that roe deer is divided into two allopatric species: the European roe deer C. capreolus, distributed over most of Europe and a few Mediterranean islands, and the Siberian roe deer C. pygargus, ranging from the Eastern Europe to the continental Asia (Lee et al., 2016). The two species are geographically separated by the physical barrier of the Urals. This implies that most of the fossil record occurring east to the Urals should be better ascribed to C. pygargus than to C. capreolus (Lister, Grubb, \& Sumner, 1998). Therefore, the ideal representation of the distribution of the fossil record ascribed to $C$. capreolus is a disjunctive geographic range, pointing to the allopatric separation between C. capreolus and C. pygargus across the Urals. We used cross validation and calculated root mean squared error (RMSE) and Area Under ROC Curve (AUC) to evaluate the goodness of fit of the model.
Lastly, we applied MInOSSE to reconstruct the geographic range of both deep time and living species: the Miocene proboscidean Deinotherium giganteum, the Jurassic ammonite Desmophyllites diphylloides, and the two living species Panthera tigris and Ursus arctos. Details about MInOSSE settings for case studies are in Appendix 5 and the full reports about Deinotherium, Desmophyllites, the tiger and the brown bear are in Appendix 8.

\section{3 | RESULTS}

\section{1 | MInOSSE performance and comparison with the other hull methods}

As regard the Eco-sims, we report here the results of all the analyses we performed by using the three scenarios for fossil localities simulation (the full list of results is available in Appendix 6). The permutational MANOVA showed there are no significant differences between MInOSSE's TSS scores when considering or not the cooccurrence analysis and this holds for all the considered scenarios (random scenario: $F=0.075, R^{2}<0.001, p=0.91$; highly clustered scenario: $F=0.128, R^{2}<0.001, p=0.836$; LGM-based scenario: $F=2.712, R^{2}=0.002, p=0.102$ ), thereby, for the sake of readability, we report the results of tests performed with co-occurrence-based MInOSSE predictions only. The ANOVA test found no significant differences between the thresholds used to binarize the RK prediction in reconstructing the actual species geographic ranges with all the cell resolutions (Supplementary Tables S2, S3 and S4 in the Appendix 6 of Supporting Information). MANOVA test found no significant differences between all the considered cell resolutions for MInOSSE predictions when considering a random ( $F=0.645$, $\left.R^{2}=0.002, p=0.656\right)$ and a clustered distribution of fossil localities $\left(F=0.561, R^{2}=0.002, p=0.721\right)$; in contrast, significant differences were found with LGM-based simulations of fossil sites $(F=5.015$, $\left.R^{2}=0.017, p=0.002\right)$. Nonetheless, the TSS scores of MInOSSE, MCP and Alpha-Hull are significantly different from each other ( $F=93.640, p \ll 0.001)$ and post-hoc results showed MInOSSE is significantly better than both MCP and Alpha-Hull with any parameters' settings, although some threshold values ('MeanProbability' and 'Observed Prevalence') proved to have a lower performance (Figure $3 a$ for the specific case of using the mean nearest neighbour distance as cell resolution, Appendix 6 and Supplementary Tables S5-S7 in Supporting Information).

As regards the performance of the three competing methods in correctly estimating the actual geographic range area, we report here the results yielded by considering the mean nearest neighbour distance as cell resolution and by using the co-occurrence analysis only. The mean values of all MInOSSE's thresholds were 2.53 times the species' actual range size, whereas the MCP and Alpha-Hull reported mean values 0.891 and 0.53 times the actual range size, respectively. By removing the worst performing thresholds, the new mean estimated area of MInOSSE is 1.961 times the actual values (see Supplementary Table S8 in Appendix 6 of Supporting Information). 
Random distribution of localities
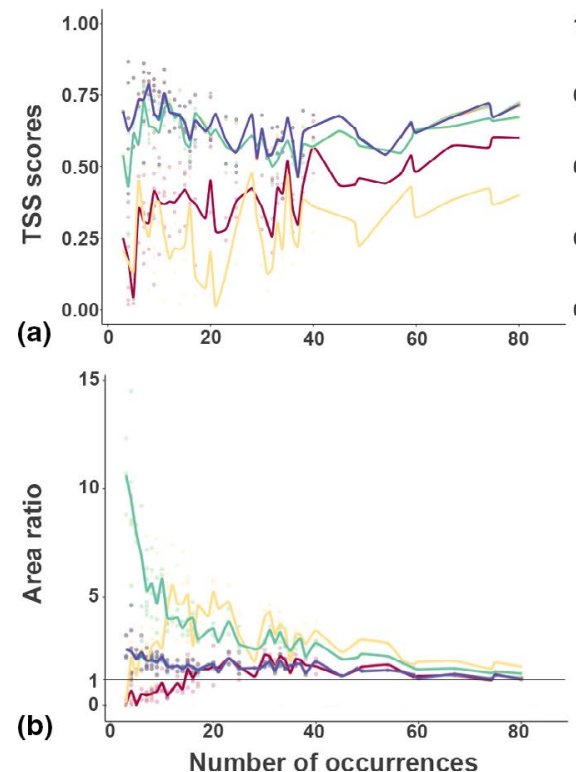

Clustered distribution of localities
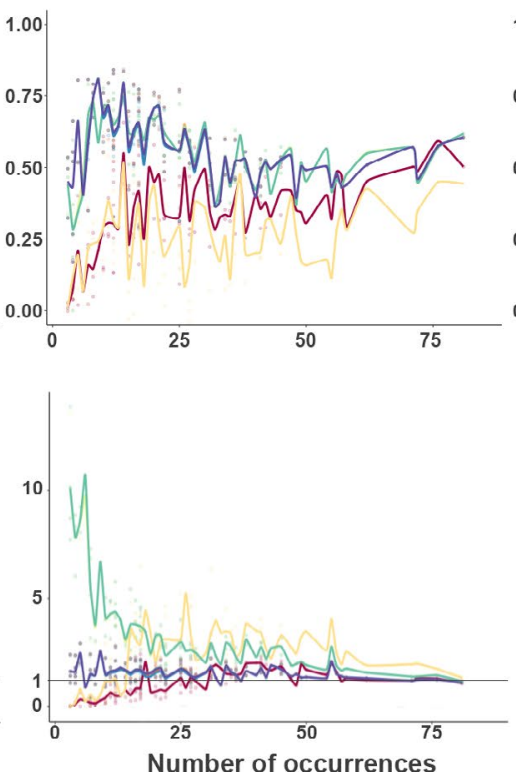

LGM-based simulations of localities
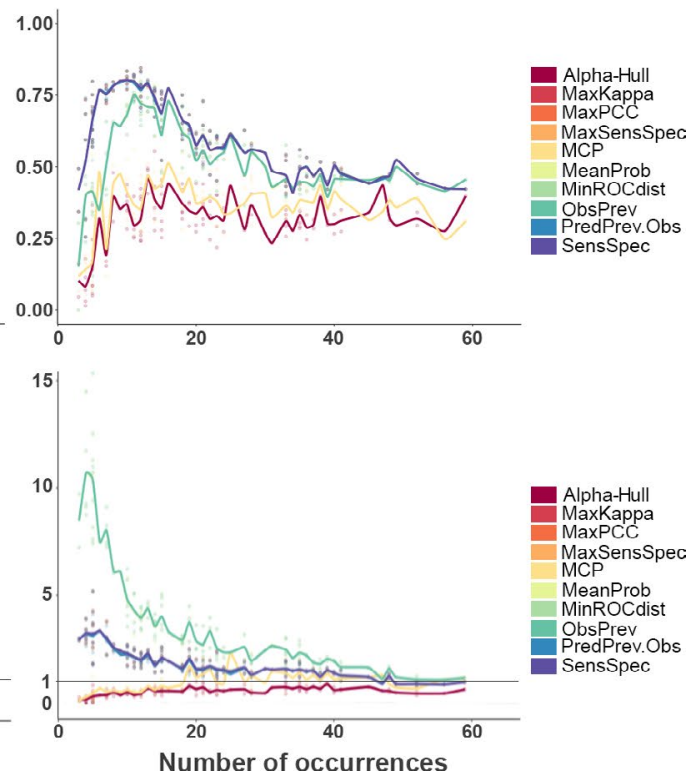

FIGURE 3 Eco-sims related results. (a) MInOSSE, minimum convex polygon (MCP) and Alpha-Hull outputs true skill statistic (TSS) scores variations with the target species' sample size when using the co-occurrence analysis. From the left, the three scenarios for fossil sites simulations: random, clustered and the last glacial maximum (LGM)-based distribution. MInOSSE predictions made by using the average nearest neighbour distance between fossil localities as raster resolution. (b) Ratios between predicted and actual surface areas of simulated species ranges. The horizontal black line at the Area ratio value 1 represents no deviation of predictions from the actual range area. Each line is built with the average true skill statistic (TSS) score or Area ratio for sample size unit and represents a method or a MInOSSE prediction with a specific threshold value. Notice that all the MInOSSE predictions are quite identical and the lines highly overlap. Points represent raw values for each sample size unit
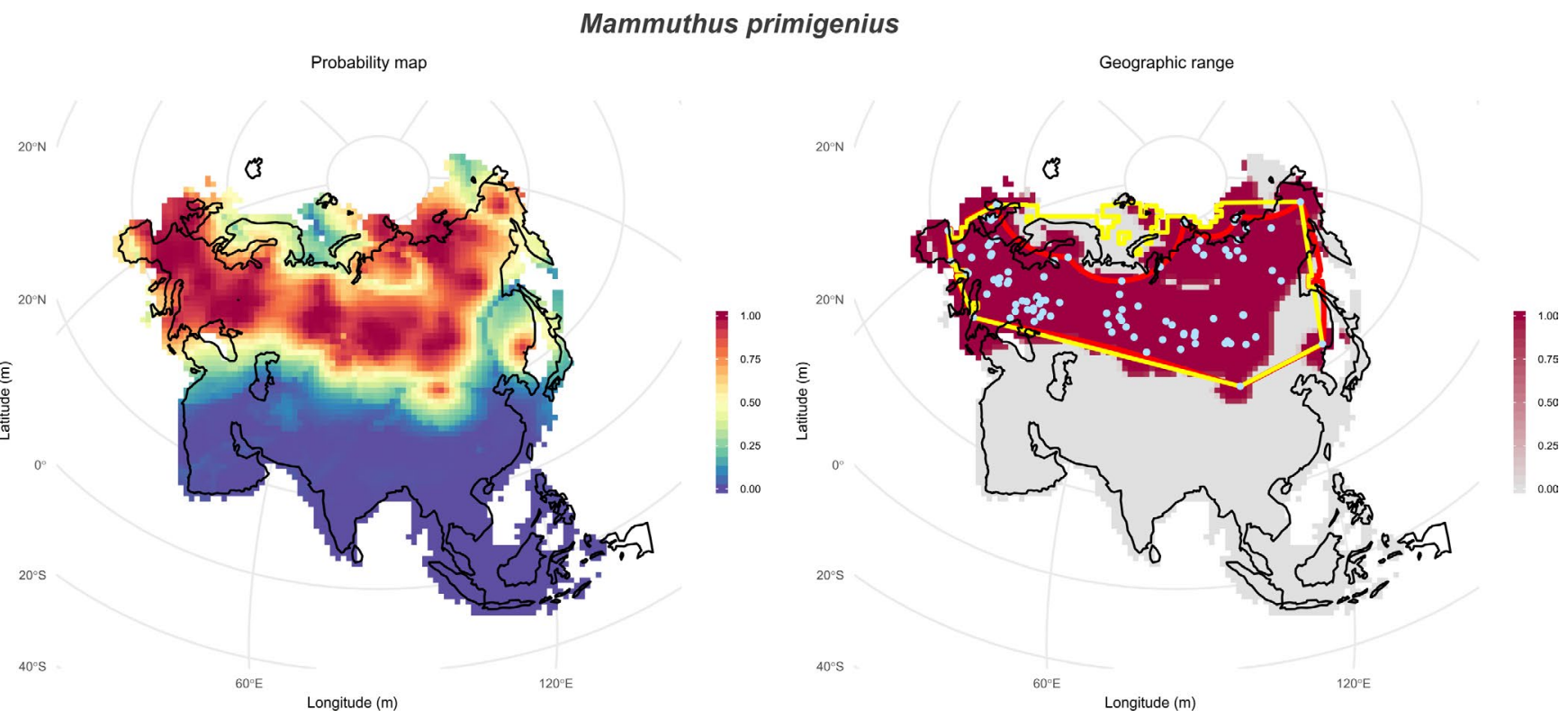

FIGURE 4 MInOSSE performed with Mammuthus primigenius fossil record ranging from 24,000 to 14,000 years ago: On the left, Regression Kriging (RK) prediction (occurrence probability); On the right, in purple: MInOSSE prediction map (MaxSensSpec threshold); in yellow, minimum convex polygon (MCP) and in red the Alpha-Hull polygon. Predictions were made by using reconstructed Eurasia continent boundaries during the last glacial maximum. Black lines show the present-day Eurasia continent boundaries. Maps showed with a Lambert Azimuthal Equal Area projection

As regards the ST-sims, when considering 100 species and 100 fossil localities, permutational MANOVA did not find significant differences between performing and not performing the co-occurrence analysis $\left(F=1.664, R^{2}=0.002, p=0.202\right)$; hence we report the results related to the use of co-occurrence analysis only. Permutational MANOVA found significant differences in the spatial clusterization 


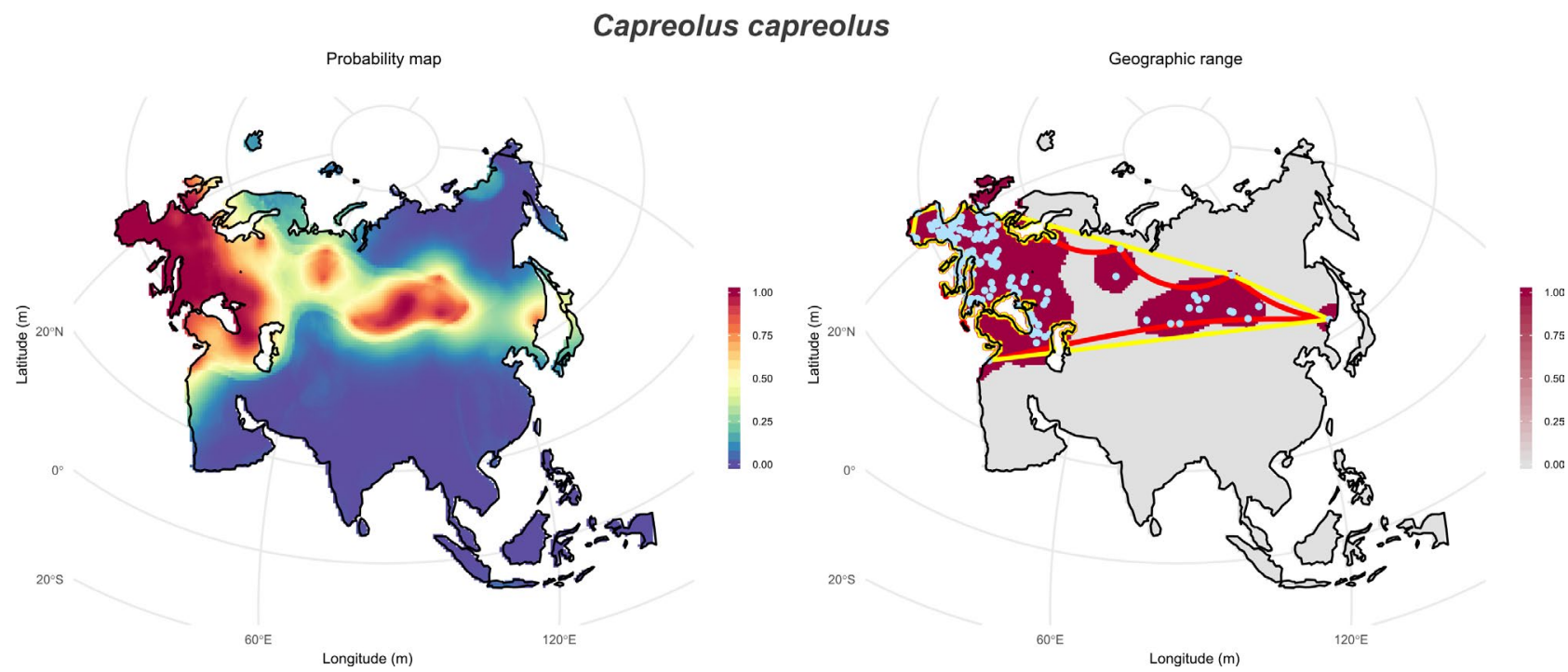

FIGURE 5 MInOSSE performed with Capreolus capreolus fossil record ranging from 130,000 to years ago to the recent. On the left, Regression Kriging (RK) prediction (occurrence probability); On the right, in purple: MInOSSE prediction map (MaxSensSpec threshold); in yellow, minimum convex polygon (MCP) and in red the Alpha-Hull polygon. Predictions were made by using present Eurasia continent boundaries (black lines). Maps showed with a Lambert Azimuthal Equal Area projection

degrees of fossil sites $\left(F=7.797, R^{2}=0.023, p=0.002\right)$, although MInOSSE's TSSs were all significantly higher than both MCP and Alpha-Hull (Supplementary Tables S9 and S11a in Appendix 6 of Supporting Information). When considering the simulations with 200 fossil localities, still no differences were found between using and not using co-occurrence analysis, and our method proved to outperform both MCP and Alpha-Hull (Appendix 6 and Supplementary Tables S10 and S11b of Supporting Information).

\subsection{Case studies}

For the reconstruction of the woolly mammoth's geographic range (Figure 4), the internal fivefold cross-validation procedure of the RK algorithm yielded an RMSE of 0.23 and an explained $79.04 \%$ of the total variation. The additional fivefold cross-validation procedure yielded an AUC value of 0.97. As we did not reduce predictors' dimensionality, we used as predictors the species that co-occurrence analysis found to be negatively related to $M$. primigenius during the LGM: Equus ferus, Rangifer tarandus, Canis lupus and Sus scrofa. As regards the case study of the roe deer (C. capreolus, Figure 5 ) record in the 130 kya to the recent time frame, the internal RK fivefold crossvalidation-related RMSE is 0.150 , whereas the explained total variance is $90.74 \%$. The additional fivefold cross-validation procedure gave AUC of 0.986 .

\section{4 | DISCUSSION}

The increasing interest in paleontological research with explicit spatial data requires developing proper tools to reconstruct fossil species' geographic ranges. These studies can make use of disparate methods, each one suited to serve specific tasks. When no environmental predictors are available to perform SDMs, polygonbased methods are well-suited for studying range size evolution and dynamics (Böhning-Gaese et al., 2006; Carotenuto, Barbera, \& Raia, 2010; Liow \& Stenseth, 2007; Raia et al., 2016; Raia, Passaro, Fulgione, \& Carotenuto, 2011; Webb \& Gaston, 2000). Maximum great circle distance is a valid tool when the study of the range size evolution is the goal (Darroch \& Saupe, 2018; Foote, Crampton, Beu, \& Cooper, 2008; Kiessling \& Aberhan, 2007; Powell, 2007). The computation of the paleolatitudinal range allows effective inferences about past species' thermal tolerance (Jablonski et al., 2013). Our simulations demonstrated that MInOSSE consistently outperforms traditional polygon-based methods in producing reliable representations of the actual spatial distributions of virtual species under a variety of conditions, sampling intensity, species diversity and with all the different parameters' settings we tested (Figure 3a). In such simulations, we took into account the climatic preferences across real fossil species in order to generate plausible geographic distributions and mimicked taphonomic biases to build artificial fossil records. In terms of TSS scores, the better performance of MInOSSE over the polygon-based methods is insensitive to the number of localities but especially evident when the number of fossil occurrences of the target species is small. This is welcome since that is the scenario when a proper approach to reconstruct the geographic range is the most demanding (Figure $3 a$ and b). Our results further show that the three methods (MInOSSE, MCP and Alpha-Hull) performances tend to converge when the number of target species' presences increases (Figure 3a). This is expected since in our simulations, the taphonomic bias is inversely related to species' abundance, hence for very abundant species, the fossil record nearly approaches the actual geographic distributions (Appendix 2 in Supporting Information). However, under all conditions, MInOSSE is far more accurate than 
the competing methods in predicting the position and shape of the geographic range (Figure 6).

When dealing with real cases, MInOSSE provided reliable results that fully comply with our expectations (Figures 4 and 5). For the woolly mammoth during the LGM, MInOSSE predictions parallel those of previous studies relying on bioclimatic variables ( $\mathrm{Di}$ Febbraro et al., 2017; Kahlke, 2015; Noguès-Bravo et al., 2008; Svenning et al., 2011). It is noteworthy that a continuous area of occurrence is predicted for the species north to Kamchatka, which is the area closest to the Bering strait, crossed by the mammoth to colonize North America. This further complies with the fossil record that documents the last stand of the woolly mammoth on the remote Eastern Siberia Wrangell Island at c. 5.7 kya (Vartanyan, Garutt, \& Sher, 1993). Northern Norway, Sweden and the Barents Sea are recognized as natural gaps in the species' geographic range, which is conceivable given they were covered by ice during the LGM and no woolly mammoth remain has ever been found there.

MInOSSE divided the roe deer fossil geographic range into different portions, east and west of the Urals. The Western portion of the range coincides almost perfectly with the current distribution of the European roe deer. The eastern range is divided into smaller, non-contiguous areas that coincide overall with the current distribution of Siberian roe deer (C. pygargus), a recent offshoot within the roe deer lineage. Thus, MInOSSE prediction complies with the emerging opinion that the easternmost fossil record traditionally ascribed to $C$. capreolus actually represents fossil Siberian roe deer occurrences. Importantly, MInOSSE applies reliably to deep time geographic distribution, where the most viable alternative, such as SDMs, cannot be used (see Supporting Information Appendix 8).

Since MInOSSE makes use of predictors, it can draw a species range even beyond the actual geographical extension of the target species' fossil record. This is a further, crucial advantage over polygon-based methods because the actual distribution of fossil sites is bound to underestimate the geographic limits of fossil species. However, a thorough quality-check of data is crucial. Indeed, although large fossil databases are now easily available for download, we stress the importance of making sure records are taxonomically consistent, and that the age and locations of fossil localities are verified. Yet, several sources of biases may occur, such as the uneven fossilization probability across species, the availability of outcrops of appropriate age and the misidentification of remains. All these factors make the fossil record spatially and temporally discontinuous, thus seriously affecting any attempt of reconstructing the past species' spatial distribution. In our method, we propose to take control of all these biases by relying on the occurrence probability of predictors species that may be evidences of the target's spatial distribution, as theorized by (Bottjer et al., 1988). Nonetheless, although MInOSSE performed better than traditional tools, our simulations can never mimic the complexity of a real fossil record, thereby some assumptions have to be meet when running MInOSSE. The first assumption is that all the involved species must have been coeval to the target one. Even in this case, in a real fossil record, there could not be a correspondence between the spatial and temporal distribution of target and predictors fossil localities, thus increasing the prediction's error (Appendix 7 in Supporting Information). Indeed, in a fossil record, the target and a predictor could appear coeval but diatopic, that is, distributed in different territories (Figure S2 in Appendix 7 of Supporting Information). On the other hand, the target and a predictor could have fossilized at the same place but in different moments of the geological history (originating a pattern of diachrony, Figure S2 in Appendix 7). The second assumption of MInOSSE is that the target and the predictors should keep constant their positive, negative or null spatial association during the considered temporal interval. It is hard to comply with this assumption when considering too long temporal windows. Stigall (2012) found Late Ordivician Brachipods were sensitive to the invasion by some other species in a way that
Random

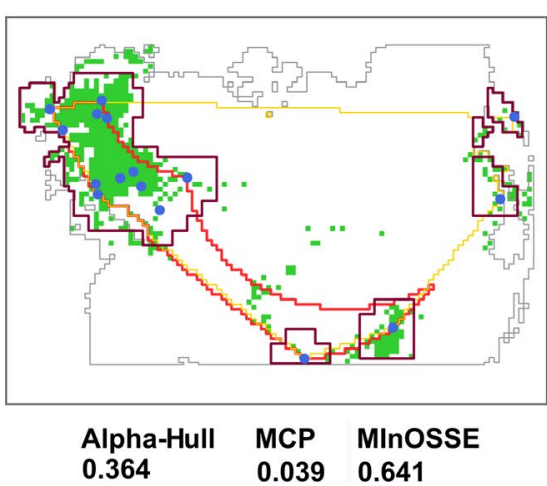

Clustered

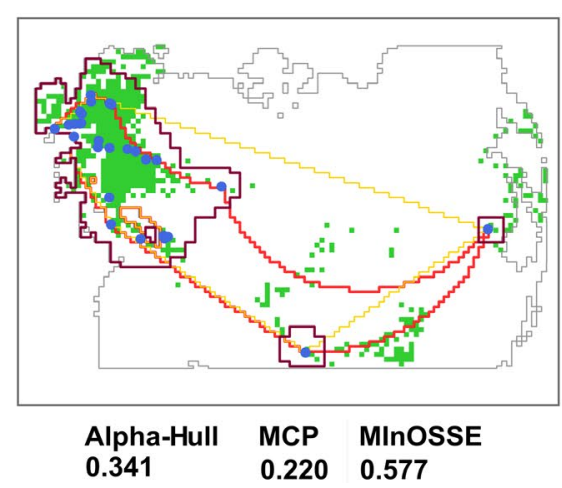

LGM-based

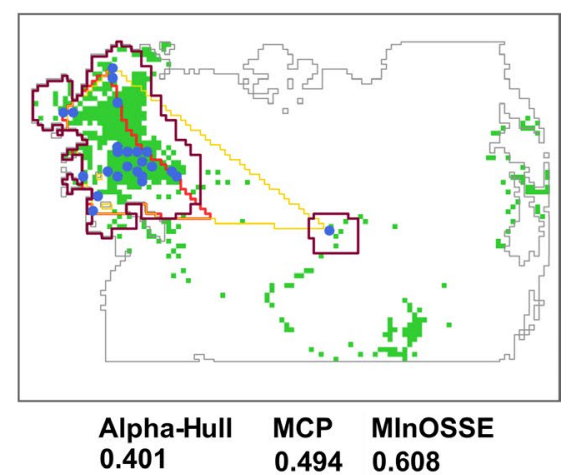

FIGURE 6 Example graphical results of spatial reconstructions with Eco-sims' fossil record. The three plots represent the different scenarios for simulating the spatial distribution of the fossil localities. From the left to the right: the random, the highly clustered and the last glacial maximum (LGM)-based spatial distribution of the simulated fossil sites. The grey-contoured polygon is the geographical domain cropped by the extent of the species' actual geographic range. The green area represents the simulated species' actual geographic distribution. The blue dots are the simulated fossil record of the considered species. The red-contoured polygon is the species' range reconstruction by the Alpha-Hull, the yellow-contoured polygon is the minimum convex polygon (MCP). The purple-contoured polygon is the MInOSSE prediction. Below each simulation plot there are the numeric values of each method's true skill statistic (TSS) scores 
they changed their ecological niche and their spatial distribution (hence ecological relationships) in a relatively short time interval. Such a case may produce misleading spatial relationships between target and predictor species, thus severely affecting the precision of a MInOSSE's prediction. A good way to circumvent a problematic situation like this is to run MInOSSE by considering shorter time frames.

All these assumptions, along with the shortcomings derived by using deep time fossil record, must be kept in mind and a very important caveat is to avoid running MInOSSE with too long temporal intervals or to consider paleo-community that showed to be reasonably stable and homogeneous through time. The use of co-occurrence analysis by means of the function minosse.data can partially fix these potential problems because it selects predictors that statistically had some relationships with the target. Users mindful of those predictors fossil localities that are spatially and temporally associated to the target species may further reduce the effect of diachrony and diatopy by specifying spatial and temporal restrictions to the predictors fossil sites in the function minosse.data (Appendix 7 in Supporting Information) as we show in the worked examples pertaining $D$. giganteum and D. diphylloides (Appendix 8 in Supporting Information).

Several methods, like MaxEnt (Phillips, Anderson, \& Schapire, 2006) and GBM (Friedman, 2001), other than MInOSSE use a model-based approach to reconstruct a species' geographic range. They share with MInOSSE the requirement of geographically explicit predictors. Such information spans from climatic variables at occurrence sites used to fit the realized niche (e.g. Guisan \& Zimmermann, 2000; Phillips et al., 2006), to complex demographical and physiological mechanisms elaborated in process-based models (e.g. Evans, Merow, Record, McMahon, \& Enquist, 2016). Some others are designed to take into account biases in detection probability by including site-occupancy models in the prediction process (MacKenzie et al., 2002). One major limitation of these approaches is that the piece of information they require is usually not available in deep past. In contrast, MInOSSE relies directly on the fossil record and can be used in time periods for which no spatially explicit information on environmental conditions is available. Moreover, MInOSSE is designed to reconstruct the actua geographic range, whereas SDMs predict environmentally suitable regions even across biogeographic barriers. With this, our method pursues essentially the same goal as a polygon-based method but provides much greater accuracy and refinement when reconstructing the shape and position of the range is the goal, rather than just the size (Figures 3 and 6).

In conclusion, by using a good-quality fossil record and complying with the most important assumptions, we argue MInOSSE is a robust and flexible model-based method for geographic range reconstruction of fossil species. We deem it will help palaeontologists opening a new window on the deep time macroecological research.

\section{ACKNOWLEDGEMENTS}

The authors declare they have no conflict of interest. We are grateful to the Senior Editor, the Associate Editor and four anonymous reviewers for all the precious suggestions for improving the method and the manuscript. This paper is dedicated to the memory of Carmela Barbera, a lovely friend and teacher.

\section{AUTHORS' CONTRIBUTIONS}

F.C. conceived the idea and designed the method; F.C., P.R., M.D.F. and A.M. conceived the simulations; F.C., S.C., C.S. and M.M. built the package; all the authors contributed to write the manuscript.

\section{DATA AVAILABILITY STATEMENT}

We provide all the MInOSSE functions as an R software (R Core Team, 2019) package at https://github.com/francesco-carotenuto/ EcoPast along with all the scripts to perform MInOSSE under both Eco-sims and ST-sims and to compare its performance with polygonbased methods: https://zenodo.org/badge/latestdoi/253762735 (Carotenuto et al., 2020).

\section{ORCID}

Francesco Carotenuto (iD https://orcid.org/0000-0001-8343-723X Mirko Di Febbraro iD https://orcid.org/0000-0001-8898-7046 Lorenzo Rook iD https://orcid.org/0000-0001-8923-5428 Pasquale Raia (iD https://orcid.org/0000-0002-4593-8006

\section{REFERENCES}

Allouche, O., Tsoar, A., \& Kadmon, R. (2006). Assessing the accuracy of species distribution models: Prevalence, kappa and the true skill statistic (TSS). Journal of Applied Ecology, 43(6), 1223-1232. https://doi. org/10.1111/j.1365-2664.2006.01214.x

Barbet-Massin, M., Jiguet, F., Albert, C. H., \& Thuiller, W. (2012). Selecting pseudo-absences for species distribution models: How, where and how many? Methods in Ecology and Evolution, 3(2), 327-338. https:// doi.org/10.1111/j.2041-210X.2011.00172.x

Böhning-Gaese, K., Caprano, T., Ewijk, K. V., \& Veith, M. (2006). Range size: Disentangling current traits and phylogenetic and biogeographic factors. The American Naturalist, 167(4), 555-567. https://doi. org $/ 10.1086 / 501078$

Bottjer, D. J., Droser, M. L., \& Jablonski, D. (1988). Palaeoenvironmental trends in the history of trace fossils. Nature, 333(6170), 252-255. https://doi.org/10.1038/333252a0

Brown, J. H. (1995). Macroecology. Chicago, IL: University of Chicago Press.

Carotenuto, F., Barbera, C., \& Raia, P. (2010). Occupancy, range size, and phylogeny in Eurasian Pliocene to recent large mammals. Paleobiology, 36(3), 399-414. https://doi.org/10.1666/09059.1

Carotenuto, F., Di Febbraro, M., Castiglione, S., Serio, C., Mondanaro, A., Melchionna, M., ... Raia, P. (2020). Data from: MInOSSE method, case studies and simulations scripts. Zenodo, https://zenodo.org/badge/ latestdoi/253762735

Carotenuto, F., Di Febbraro, M., Melchionna, M., Castiglione, S., Saggese, F., Serio, C., ... Raia, P. (2016). The influence of climate on species distribution over time and space during the late Quaternary. Quaternary Science Reviews, 149, 188-199. https://doi.org/10.1016/j.quasc irev.2016.07.036

Carotenuto, F., Di Febbraro, M., Melchionna, M., Mondanaro, A., Castiglione, S., Serio, C., ... Raia, P. (2018). The well-behaved killer: Late Pleistocene humans in Eurasia were significantly associated with living megafauna only. Palaeogeography, Palaeoclimatology, Palaeoecology, 500, 24-32. https://doi.org/10.1016/j.palaeo.2018.03.036

Carotenuto, F., Diniz-Filho, J. A. F., \& Raia, P. (2015). Space and time: The two dimensions of Artiodactyla body mass evolution. 
Palaeogeography, Palaeoclimatology, Palaeoecology, 437, 18-25. https://doi.org/10.1016/j.palaeo.2015.07.013

Chen, T., \& Guestrin, C. (2016, August). Xgboost: A scalable tree boosting system. In Proceedings of the 22nd ACM SIGKDD International Conference on Knowledge Discovery and Data Mining (pp. 785-794). New York, NY: Association for Computing Machinery. https://doi. org/10.1145/2939672.2939785

Darroch, S. A., \& Saupe, E. E. (2018). Reconstructing geographic rangesize dynamics from fossil data. Paleobiology, 44(1), 25-39. https://doi. org/10.1017/pab.2017.25

Di Febbraro, M., Carotenuto, F., Castiglione, S., Russo, D., Loy, A., Maiorano, L., \& Raia, P. (2017). Does the jack of all trades fare best? Survival and niche width in Late Pleistocene megafauna. Journal of Biogeography, 44(12), 2828-2838. https://doi.org/10.1111/jbi.13078

Duan, R. Y., Kong, X. Q., Huang, M. Y., Wu, G. L., \& Wang, Z. G. (2015). SDMvspecies: A software for creating virtual species for species distribution modelling. Ecography, 38(1), 108-110. https://doi. org/10.1111/ecog.01080

Evans, M. E., Merow, C., Record, S., McMahon, S. M., \& Enquist, B. J. (2016). Towards process-based range modeling of many species. Trends in Ecology \& Evolution, 31(11), 860-871. https://doi. org/10.1016/j.tree.2016.08.005

Foote, M., Crampton, J. S., Beu, A. G., \& Cooper, R. A. (2008). On the bidirectional relationship between geographic range and taxonomic duration. Paleobiology, 34(4), 421-433. https://doi.org/10.1666/08023.1

Freeman, E. A., \& Moisen, G. (2008). PresenceAbsence: An R package for presence absence analysis. Journal of Statistical Software, 23(11), 31. https://doi.org/10.18637/jss.v023.i11

Friedman, J. H. (2001). Greedy function approximation: A gradient boosting machine. Annals of Statistics, 29(5), 1189-1232. https://doi. org/10.1214/aos/1013203451

Friedrich, T., Timmermann, A., Menviel, L., Timm, O. E., Mouchet, A., \& Roche, D. M. (2010). The mechanism behind internally generated centennial-to-millennial scale climate variability in an earth system model of intermediate complexity. Geoscientific Model Development, 3(2), 377. https://doi.org/10.5194/gmd-3-377-2010

Gaston, K., \& Blackburn, T. (2000). Pattern and process in macroecology. Malden, MA: Wiley-Blackwell. https://doi.org/10.1002/97804 70999592

Gaston, K. J., \& Curnutt, J. L. (1998). The dynamics of abundance-range size relationships. Oikos, 81(1), 38-44. https://doi.org/10.2307/3546465

Gotelli, N. J., \& McCabe, D. J. (2002). Species co-occurrence: A meta-analysis of JM Diamond's assembly rules model. Ecology, 83(8), 2091-2096. https://doi.org/10.1890/0012-9658(2002)083[2091:SCOAMA]2.0.CO;2

Griffith, D. M., Veech, J. A., \& Marsh, C. J. (2016). Cooccur: Probabilistic species co-occurrence analysis in R. Journal of Statistical Software, 69(2), 1-17. https://doi.org/10.18637/jss.v069.c02

Guisan, A., \& Zimmermann, N. E. (2000). Predictive habitat distribution models in ecology. Ecological Modelling, 135(2-3), 147-186. https:// doi.org/10.1016/S0304-3800(00)00354-9

Guthrie, R. D. (1982). Mammals of the mammoth steppe as paleoenvironmental indicators. In Paleoecology of Beringia (pp. 307-326). New York, NY: Academic Press. https://doi.org/10.1016/B978-0-12355860-2.50030-2

Hengl, T. (2020). GSIF: Global soil information facilities. R package version 0.5-5.1. Retrieved from https://CRAN.R-project.org/package=GSIF

Hengl, T., Heuvelink, G. B. M., Kempen, B., Leenaars, J. G. B., Walsh, M. G., Shepherd, K. D., ... Tondoh, J. E. (2015). Mapping soil properties of Africa at $250 \mathrm{~m}$ resolution: Random forests significantly improve current predictions. PLOS ONE, 10(6), e0125814. https://doi. org/10.1371/journal.pone.0125814

Hengl, T., Heuvelink, G. B., \& Rossiter, D. G. (2007). About RegressionKriging: From equations to case studies. Computers \& Geosciences, 33(10), 1301-1315. https://doi.org/10.1016/j.cageo.2007.05.001
Hengl, T., Mendes de Jesus, J., Heuvelink, G. B. M., Ruiperez Gonzalez, M., Kilibarda, M., Blagotić, A., ... Kempen, B. (2017). SoilGrids250m: Global gridded soil information based on machine learning. PLOS ONE, 12(2), e0169748. https://doi.org/10.1371/journal.pone.0169748

Hengl, T., Sierdsema, H., Radović, A., \& Dilo, A. (2009). Spatial prediction of species' distributions from occurrence-only records: Combining point pattern analysis. ENFA and Regression-Kriging. Ecological Modelling, 220(24), 3499-3511. https://doi.org/10.1016/j.ecolmodel.2009.06.038

Hortal, J., de Bello, F., Diniz-Filho, J. A. F., Lewinsohn, T. M., Lobo, J. M., \& Ladle, R. J. (2015). Seven shortfalls that beset large-scale knowledge of biodiversity. Annual Review of Ecology, Evolution, and Systematics, 46, 523-549. https://doi.org/10.1146/annurev-ecolsys-112414-054400

Jablonski, D. (1987). Heritability at the species level: Analysis of geographic ranges of Cretaceous mollusks. Science, 238(4825), 360363. https://doi.org/10.1126/science.238.4825.360

Jablonski, D., Belanger, C. L., Berke, S. K., Huang, S., Krug, A. Z., Roy, K., ... Valentine, J. W. (2013). Out of the tropics, but how? Fossils, bridge species, and thermal ranges in the dynamics of the marine latitudinal diversity gradient. Proceedings of the National Academy of Sciences of the United States of America, 110(26), 10487-10494. https://doi. org/10.1073/pnas.1308997110

Kahlke, R. D. (2015). The maximum geographic extension of Late Pleistocene Mammuthus primigenius (Proboscidea, Mammalia) and its limiting factors. Quaternary International, 379, 147-154. https://doi. org/10.1016/j.quaint.2015.03.023

Kiessling, W., \& Aberhan, M. (2007). Geographical distribution and extinction risk: Lessons from Triassic-Jurassic marine benthic organisms. Journal of Biogeography, 34(9), 1473-1489. https://doi. org/10.1111/j.1365-2699.2007.01709.x

Lee, Y. S., Markov, N., Argunov, A., Voloshina, I., Bayarlkhagva, D., Kim, B. J., ... Kim, K. S. (2016). Genetic diversity and phylogeography of Siberian roe deer, Caproulus pygargus, in central and peripheral populations. Ecology and Evolution, 6(20), 7286-7297. https://doi. org/10.1002/ece3.2458

Liow, L. H., \& Stenseth, N. C. (2007). The rise and fall of species: Implications for macroevolutionary and macroecological studies. Proceedings of the Royal Society B: Biological Sciences, 274(1626), 2745-2752. https://doi.org/10.1098/rspb.2007.1006

Lister, A. M., Grubb, P., \& Sumner, S. R. M. (1998). Taxonomy, morphology and evolution of European roe deer. In R. Andresen, P. Duncan, \& J. D. C. Linnel (Eds.), The European roe deer (pp. 23-46). Oslo, Norway: Scandinavian University Press.

MacKenzie, D. I., Nichols, J. D., Lachman, G. B., Droege, S., Andrew Royle, J., \& Langtimm, C. A. (2002). Estimating site occupancy rates when detection probabilities are less than one. Ecology, 83(8), 2248-2255. https://doi.org/10.1890/0012-9658(2002)083[2248:ESORW D]2.0.CO;2

Melchionna, M., Di Febbraro, M., Carotenuto, F., Rook, L., Mondanaro, A., Castiglione, S., ... Raia, P. (2018). Fragmentation of Neanderthals' pre-extinction distribution by climate change. Palaeogeography, Palaeoclimatology, Palaeoecology, 496, 146-154. https://doi.org/10.1016/ j.palaeo.2018.01.031

Montgomery, D. C. (2004). Design and analysis of experiments 6th edition with design expert software. Hoboken, NJ: John Wiley \& Sons.

Nogués-Bravo, D., Rodríguez, J., Hortal, J., Batra, P., \& Araújo, M. B. (2008). Climate change, humans, and the extinction of the woolly mammoth. PLoS Biology, 6(4), e79. https://doi.org/10.1371/journal.pbio.0060079

Noto, C. R. (2011). Hierarchical control of terrestrial vertebrate taphonomy over space and time: Discussion of mechanisms and implications for vertebrate paleobiology. In I. P. A. Allison \& D. J. Bottjer (Eds.), Taphonomy. Aims \& scope topics in geobiology book series (pp. 287-336). Dordrecht, the Netherlands: Springer. https://doi. org/10.1007/978-90-481-8643-3_8

Pebesma, E., Cornford, D., Dubois, G., Heuvelink, G. B., Hristopulos, D., Pilz, J., ... Skøien, J. O. (2011). INTAMAP: The design and 
implementation of an interoperable automated interpolation web service. Computers \& Geosciences, 37(3), 343-352. https://doi. org/10.1016/j.cageo.2010.03.019

Phillips, S. J., Anderson, R. P., \& Schapire, R. E. (2006). Maximum entropy modelling of species geographic distributions. Ecological Modelling 190(3-4), 231-259. https://doi.org/10.1016/j.ecolmodel.2005.03.026

Plotnick, R. E. (2017). Recurrent hierarchical patterns and the fractal distribution of fossil localities. Geology, 45(4), 295-298. https://doi. org/10.1130/G38828.1

Powell, M. G. (2007). Geographic range and genus longevity of late Paleozoic brachiopods. Paleobiology, 33, 530-546. https://doi. org/10.1666/07011.1

Preston, F. W. (1962). The canonical distribution of commonness and rarity: Part I. Ecology, 43(2), 185-215. https://doi.org/10.2307/1931976

R Core Team. (2019). R: A language and environment for statistical computing. Vienna, Austria: R Foundation for Statistical Computing. Retrieved from https://www.R-project.org/

Raia, P., Carotenuto, F., Meloro, C., Piras, P., Barbera, C., \& Kotsakis, T. (2009). More than three million years of community evolution. The temporal and geographical resolution of the Plio-Pleistocene Western Eurasia mammal faunas. Palaeogeography, Palaeoclimatology, Palaeoecology, 276(1-4), 15-23. https://doi.org/10.1016/j.palaeo.2009.02.005

Raia, P., Carotenuto, F., Mondanaro, A., Castiglione, S., Passaro, F., Saggese, F., ... Fortelius, M. (2016). Progress to extinction: Increased specialisation causes the demise of animal clades. Scientific Reports, 6, 30965. https://doi.org/10.1038/srep30965

Raia, P., Passaro, F., Fulgione, D., \& Carotenuto, F. (2011). Habitat tracking, stasis and survival in Neogene large mammals. Biology Letters, 8 , 64-66. https://doi.org/10.1098/rsbl.2011.0613

Singarayer, J. S., \& Valdes, P. J. (2010). High-latitude climate sensitivity to ice-sheet forcing over the last $120 \mathrm{kyr}$. Quaternary Science Reviews, 29(1-2), 43-55. https://doi.org/10.1016/j.quascirev.2009.10.011

Stigall, A. L. (2012). Using ecological niche modelling to evaluate niche stability in deep time. Journal of Biogeography, 39(4), 772-781. https://doi.org/10.1111/j.1365-2699.2011.02651.x

Svenning, J. C., Fløjgaard, C., Marske, K. A., Nógues-Bravo, D., \& Normand, S. (2011). Applications of species distribution modelling to paleobiology. Quaternary Science Reviews, 30(21-22), 2930-2947. https://doi.org/10.1016/j.quascirev.2011.06.012
Varela, S., Anderson, R. P., García-Valdés, R., \& Fernández-González, F. (2014). Environmental filters reduce the effects of sampling bias and improve predictions of ecological niche models. Ecography, 37(11), 1084-1091. https://doi.org/10.1111/j.1600-0587.2013.00441.x

Varela, S., Lobo, J. M., \& Hortal, J. (2011). Using species distribution models in paleobiogeography: A matter of data, predictors and concepts. Palaeogeography, Palaeoclimatology, Palaeoecology, 310(3-4), 451-463. https://doi.org/10.1016/j.palaeo.2011.07.021

Vartanyan, S. L., Garutt, V. E., \& Sher, A. V. (1993). Holocene dwarf mammoths from Wrangel Island in the Siberian Arctic. Nature, 362(6418), 337. https://doi.org/10.1038/362337a0

Webb, T. J., \& Gaston, K. J. (2000). Geographic range size and evolutionary age in birds. Proceedings of the Royal Society of London. Series B: Biological Sciences, 267(1455), 1843-1850. https://doi.org/10.1098/ rspb.2000.1219

Whittaker, R. J., \& Fernández-Palacios, J. M. (2007). Island biogeography: Ecology, evolution, and conservation. Oxford, UK: Oxford University Press.

Youden, W. J. (1950). Index for rating diagnostic tests. Cancer, 3(1), 3235. https://doi.org/10.1002/1097-0142(1950)3:1<32:AID-CNCR2 $820030106>3.0 . C O ; 2-3$

\section{SUPPORTING INFORMATION}

Additional supporting information may be found online in the Supporting Information section.

How to cite this article: Carotenuto F, Di Febbraro M, Mondanaro A, et al. MInOSSE: A new method to reconstruct geographic ranges of fossil species. Methods Ecol Evol. 2020;11:1121-1132. https://doi.org/10.1111/2041$210 \times .13423$ 